Rodrigues K.H. e Colaboradoras - A Enfermagem no Projeto de Cooperação Educacional Vila Paranoá. Rev. Bras. Enf.; RS.36: 183 - 192, 1983.

possibilitou desenvolver nos alunos experiências de trabalho com a comunidade e família carentes; por outro lado, com o prosseguimento do projeto esperamos poder em breve atingir as propostas de ação e cobertura a que nos propusemos.

Desta forma estaremos dando aos alunos do Curso de Enfermagem e Nutrição uma visão ampla da realidade do campo profissional que ele poderá atuar no futuro: experiência de comunidade, experiência nos Serviços de Assistência dos Postos de Saúde da Secretaria de Saúde, e experiência hospitalar.

\title{
BIBLIOGRAFIA
}

1. Andrade, M. N.; Lima, M. L. F. - "O Projeto Vitória - A experiência da Universidade Federal de Pernambuco" - Anais do XXX Congresso Brasileiro de Enfermagem - ABEn - Belém, 16 a 22 de julho de 1978;

2. Duarte/Muxfeldt - Saúde Materna, Hospital de Clínicas de Porto Alegre, Porto Alegre-RS, 1976;

3. Nogueira, Maria Jacyra de Campos et al - Manual para o ensino de Enfermagem de Saúde Pública, São Paulo: Sociedade Beneficente São Camilo, 1978;

4. Paim, L; Costa, L. A. T.; Wright, M. G. M. - "As inovações no Ensino Superior de Enfermagem, face a assistência à saúde da população - Possibilidades e Limitações" - Anais do XXX Congresso Brasileiro de Enfermagem - ABEn - Belém, 16 a 22 de julho de 1978;

5. Ministério da Saúde, Secretaria de Saúde Pública, Divisão Nacional de Organização Sanitária, Setor de Enfermagem - Manual para Programação de Penetração Rural - Rio de Janeiro, 1974;

6. Ministério da Saúde, Secretaria Nacional de Ações Básicas de Saúde, Divisão Nacional de Organização de Serviços de Saúde, Manual para Posto de Saúde - Documento Preliminar - Brasília, 1981;

7. Vieira, César Augusto de Barros - "Extensão de cobertura no Brasil: crise e reforma do sistema de saúde" - Anais do XXX Congresso Brasileiro de Enfermagem - ABEn - Belém 16 a 22 de julho de 1978.

\section{POPULAÇÃO DOS ESTADOS DA REGIÃO SUL DO BRASIL E O COMPROMISSO SOCIAL DA ENFERMAGEM COM O IDOSO}

\author{
* Maria de Lourdes de Souza \\ ** Jonas Salomão Spriccigo
}

ReBEn/08

Souza M. L. e Colaborador - População dos Estados da Região Sul do Brasil e o Compromisso Social da Enfermagem com o Idoso Rev. Bras. Enf.; RS, 36: 192 - 199, 1983

\section{RESUMO}

Os autores apresentam considerações teóricas acerca de questões sociais do envelhecimento individual e populacional. Estabelecem comparações entre os grupos etários de menores de 20 anos e maiores de 60 anos, da população do Brasil, destacando os Estad̦os do Rio Grande do Sul, Santa Catarina e Paraná. Referem os conceitos que fundamentam a assistência de enferrnagem e criticam sua prática na assistência ao idoso. Concluem que a enfermagem deve participar do processo de educação so-

* Doutor em Saúde Pública e Professor Adjunto do Departamento de Saúde Pública do Centro de Ciências'da Saúde da Universidade Federal de Santa Catarina.

** Professor Assistente do Departamento de Enfermagem do Centro de Ciências da Saúde da Universidade Federal de Santa Catarina. 
Souza M.L. e Colaborador - População dos Estados da Região Sul do Brasil e o Compromisso Social da Enfermagem com o Idoso. Rev. Bras. Enf.; RS.36: 192 - 199, 1983.

cial, buscando maior valorização do ser humano em idade avançada. Consideram ainda, necessária a reavaliação da prática da enfermagem, de modo que a assistência não seja mais um instrumento de anulação do ser humano em idade avançada e sim, favoreça a libertação dos mitos impostos pela sociedade.

\section{INTRODUÇÃO}

QUADRA14 diz que "segundo estimativas grosseiras, o homem está na face da terra há uns 600 mil anos... Em todo esse período de tempo, absolutamente inalcançável pelo raciocínio lógico, o fenômeno velhice é algo paradoxalmente novo". Acrescenta ainda que, "apesar de sua modernidade, a velhice não deixou de ser incluida por OZBERKHAN entre os problemas que denominou de críticos contínuos: universais, inquestionáveis, carentes de estudos multidisciplinares".

A velhice sendo parte constituinte de direito e de fato da vida do ser humano, assume grande importância a medida em que se relaciona fortemente com as condiçōes sócio-econômicas em especial quando estas forem adversas. Esta afirmativa carreia consigo vários aspectos a serem abordados, no entanto os autores decidiram enfocar no presente trabalho, algumas questões sociais do idoso e sua relação com a prática da enfermagem, a nível de envelhecimento individual e populacional.

Segundo THOMAS16 "o envelhecimento individual põe em destaque etapas irreversíveis do ciclo de vida, cuja compreensão depende do conhecimento dos processos biológicos, sociológicos e psicológicos associados ao crescimento, amadurecimento e possível declínio do organismo humano, no contexto de ambiente físico, psicológico e social". Quanto ao envelhecimento populacional, acrescenta este autor que "constitui um fenômeno mais complexo, em relação ao qual pouco consenso existe no que se refere a como pode ser definida. Grande parte da literatura existente até agora, ao definir o envelhecimento social, põe em destaque o incremento proporcional do número de pessoas mais velhas dentro de cada população".

$\mathrm{Na}$ tentativa de relacionar estes conceitos a responsabilidade social da enfermagem com o idoso, apresenta-se a população brasileira e dos Estados da Região Sul enfocando os grupos etários de menores de 20 e maiores de 60 anos. Basicamente este trabalho aponta questionamentos acerca da realidade social do idoso e sua relação com a prática'da enfermagem.

\section{DISTRIBUIÇÃO DA POPULAÇÃO POR GRUPO ETÁRIO E ESPERANÇA DE VIDA}

Os dados do Quadro I permitem verificar a evolução da população do Brasil e dos Estados da Região Sul segundo os grupos etários selecionados para efeitos comparativos. No período de $1960 \mathrm{a}$ 1980, observa-se que no grupo dos menores de 20 anos houve no Brasil uma redução de $8,18 \%$, no Rio Grande do Sul de $15,16 \%$, em Santa Catarina de $16,58 \%$ e no Paraná $8,91 \%$. No grupo de maiores de 60 anos, no mesmo período, no Brasil verifica-se $\iota m$ incremento de $36,57 \%$, no Rio Grande do Sul, $44,42 \%$, em Santa Catarina $45,48 \%$ e no Paraná $59,10 \%$.

Os dados relativos ao grupo da população com 60 e mais anos parecem muito representativos quando comparados com os que refere FUSTINONI 9 , "na França em 1966, 13,5\% da população tinha mais de 65 anos, e nos Estados Unidos em 1978, o número de pessoas com mais de 65 anos era de 20 milhões". QUADRA ${ }^{14}$ diz que "no final da década de 60 , a Āustria, Bélgica, França, Itália, Países Baixos, República Federal da Alemanha e Suiça contavam com uma participação percentual da população de 65 e mais, superior a $10 \%$, alcançando no primeiro desses países cifras de $14 \%$ ". Complementa ainda a interpretação dos dados do Quadro 1, o que THOMAS16 cita "a população de 60 anos ou mais, embora representasse apenas $8,4 \%$ da população total do mundo em 1970 e deva representar $9,3 \%$ no ano 2000 , se traduz em números absolutos em, respectivamente, 304 milhões e 581 milhões de pessoas. Essa quase duplicação dos grupos de idade mais avançada no prazo.de 30 anos tem conseqüências bem maiores do que sugeririam suas percentagens das populações totais".

A apresentação do segundo grupo etário e m 60 e mais anos praticamente foi determinado pelas informações registradas na FIBGE 11 acerca da esperança de vida ao nascer que no Brasil no período de 1960/1970 ęra de 54,9 anos para sexo masculino e 59,0 anos para o feminino; entre 1970/1975 era de 58,8 anos para o sexo masculino e 63,1 anos para o feminino (somente aí é que se observa expectativa de vida superior a 60 anos), e no período de $1975 / 1980$ é de 61,3 anos para o sexo masculino e 65,5 anos para o sexo feminino. Estas informações comparadas as do Quadro 2, as de SVANBORG ${ }^{15}$ que refere ser a expectativa de vida ao nascer de 73 anos para os homens e 77 anos para as mulheres na Suécia, e, as de VIEDMA ${ }^{18}$ que diz, que a vida média do homem americano que era de 49 anos em 1900 , 
Souza M.L. e Colaborador - População dos Estados da Região Sul do Brasil e o Compromisso Social da Enfermagem com o Idoso. Rev. Bras. Enf.; RS.36: 192 - 199, 1983.

hoje em dia é de cerca de 70 anos. Parece-nos que estas informações quando comparadas entre si, podem a priori demonstrar uma diferenciação da esperança de vida segundo as condições de desenvolvimento do país. Condições de desenvolvimento estas que podem ser avaliadas de inúmeras formas, uma delas é a renda mensal familiar, por isto é que se segue a Tabela 1.

QUADRO 1 - Distribuição do número e percentagem da população dos Estados da Região Sul e do Brasil, segundo grupo etário e ano.

\begin{tabular}{|c|c|c|c|c|c|c|c|c|c|}
\hline \multirow{2}{*}{ Ano } & & \multicolumn{2}{|c|}{ BRASIL } & \multicolumn{2}{|c|}{ PARANÁ } & \multicolumn{2}{|c|}{ SANTA CATARINA } & \multicolumn{2}{|c|}{ RIO GRANDE DO SUL } \\
\hline & ETÁRIO & N. & $\%$ & N. & $\%$ & N. & $\%$ & N. & $\%$ \\
\hline \multirow{2}{*}{1960} & 20 anos & 37.024 .746 & 52,83 & 2.361 .049 & 55,31 & 1.331 .536 & 59,52 & 2.781 .215 & 50,65 \\
\hline & 60 anos & 3.312 .420 & 4,73 & 142.995 & 3,35 & 86.755 & 3,87 & 274.684 & 5,11 \\
\hline \multirow{2}{*}{1970} & 20 anos & 49.383 .716 & 53,02 & 3.916 .914 & 56,52 & 1.642 .218 & 56,58 & 3.347 .054 & 50,22 \\
\hline & 60 anos & 4.716 .208 & 5,06 & 254.495 & 3,67 & 131.261 & 4,53 & 384.502 & 5,77 \\
\hline \multirow{2}{*}{1980} & 20 anos & 57.748 .242 & 48,51 & 3.836 .413 & 50,28 & 1.803 .098 & 49,65 & 3.342 .323 & 42,97 \\
\hline & 60 anos & 7.699 .067 & 6,46 & 407.123 & 5,33 & 204.588 & 5,63 & 573.933 & 7,38 \\
\hline
\end{tabular}

FONTE: F. IBGE 10 Registros censitários

TABELA 1 - Estimativas de esperança de vida ao nascer segundo grupos de rendimento mensal familiar. Brasil/1976.

\section{RENDIMENTO MENSAL FAMILIAR}

ESPERANÇA DE VIDA AO

NASCER ESTIMADA

Até 1 salário mínimo

54,8 anos

Mais de 1 a 2 salários mínimos

59,5 anos

Mais de 2 a 5 salários mínimos

64,0 anos

Mais de 5 salários mínimos

69,6 anos

Todos os níveis

60,5 anos

Fonte: F. IBGE 11 
Souza M.L. e Colaborador - População dos Estados da Região Sul do Brasil e o Compromisso Social da Enfermagem com o Idoso. Rev. Bras. Enf.; RS.36: 192 - 199, 1983.

Quadro 2 - Esperança de vida ao nascer em alguns países e em Santa Catarina - 1974 ou anos próximos.

\begin{tabular}{|c|c|c|c|}
\hline PAÍSES & ANO & HOMENS & MULHERES \\
\hline Bolívia & $1970-75$ & 45,7 & 47,9 \\
\hline El Salvador & 1973 & 61,2 & 66,8 \\
\hline México & 1973 & 63,9 & 67,0 \\
\hline Chile & 1973 & 60,6 & 67,8 \\
\hline SANTA CATARINA & $1975-76$ & 63,3 & 69,2 \\
\hline Argentina & $1970-75$ & 65,2 & 71,4 \\
\hline Iugoslávia & 1974 & 67,0 & 72,0 \\
\hline Hungria & 1975 & 66,4 & 72,5 \\
\hline Portugual & 1975 & 65,1 & 72,6 \\
\hline Costa Rica & 1974 & 69,2 & 73,4 \\
\hline Bulgária & 1975 & 68,7 & 73,5 \\
\hline Israel (pop. jud:) & 1974 & 70,5 & 73,7 \\
\hline Rep. Dem. da Alemanha & 1975 & 68,6 & 74,1 \\
\hline Cuba & 1974 & 71,2 & 74,2 \\
\hline Áustria & 1975 & 67,6 & 74,7 \\
\hline Rep. Fed. da Alemanha & 1974 & 68,2 & 74,7 \\
\hline Nova Zelândia & 1974 & 68,9 & 75,0 \\
\hline Austrália & 1974 & 68,0 & 75,1 \\
\hline Bélgica & 1974 & 68,7 & 75,2 \\
\hline Estados Unidos & 1974 & 68,2 & 76,0 \\
\hline Itália & 1974 & 69,9 & 76,1 \\
\hline Espanha & 1974 & 70,6 & 76,2 \\
\hline Grécia & 1974 & 72,5 & 76,9 \\
\hline Canadá & 1974 & 69,6 & 77,1 \\
\hline Japão & 1975 & 71,9 & 77,2 \\
\hline Dinamarca & 1975 & 71,5 & 77,2 \\
\hline França & 1974 & 69,5 & 77,6 \\
\hline Noruega & 1973 & 71,4 & 77,9 \\
\hline Suécia & 1975 & 72,2 & 78,1 \\
\hline Suiça & 1975 & 71,6 & 78,2 \\
\hline Islândia & 1975 & 72,3 & 79,0 \\
\hline
\end{tabular}

Fonte: BARROS, Filho ${ }^{1}$. Contribuição ao estudo da mortalidade no Estado de Santa Catarina. Florianópolis, 1979.

Pelos dados desta tabela, observa-se que os de maior renda tem maior esperança de vida. Entre os que tem renda mensal familiar de menos de 1 salário mínimo há uma perda de 14,8 anos em relação aos que tem rendimento superior a 5 salários mínimos. Na Região Sul ainda segundo a FIBGE ${ }^{11}$, esta tendência é observável uma vez que no ano de 1970 o grupo de menor renda tinha esperanças de vida de 60,5 anos e para os de renda mais alta 66,9 anos.

Esta situação se agrava se atentarmos para o fato de que a aposentadoria é legislada para os 65 anos, e muito mais grave se configura se relacionarmos ao fato de que dos brasileiros economicamente ativos $37,4 \%$ percebiam no ano de 1976 até 1 salário mínimo e $66,8 \%$ com até 2 salários mínimos.

Como se isto não bastasse para configurar um dos aspectos básicos da situação em que provavelmente a população de idosos é submetida, em 1973 no Brasil somente 4,9\% da população economicamente ativa tinha mais de 60 anos, e em 1976 somente 4,5\%. Estes dados vão ao encontro do que refere $\operatorname{COSTA}^{8}$ "o principal determinante da qualidade da vida da pessoa idosa é o seu nível de renda". Acrescenta ainda "a maioria esmagadora dos idosos, no Brasil e em outros países menos desenvolvidos, depende de familiares, da caridade pública ou religiosa, da assistência estatal ou de magras pensões, geralmente 
Souza M.L. e Colaborador - População dos Estados da Região Sul do Brasil e o Compromisso Social da Enfermagem com o Idoso. Rev. Bras. Enf.; RS.36: 192 - 199, 1983.

corroídas pela inflação endêmica dos dias atuais, em todos os países. Seu poder de compra, quando o tem, é diminuto, mal dando para atender as necessidades básicas. Assim, a qualidade da vida de que desfrutam deixa muitíssimo a desejar".

\section{NECESSIDADES HUMANAS BÁSICAS DO IDOSO E CONFLITOS COM METAS SOCIAIS}

Os direitos das pessoas idosas foram consagradas em muitas cartas, uma das quais é nada menos que a Declaração Universal dos Direitos Humanos, que proclamou especificamente isso há 30 anos - o conceito de bem estar e segurança financeira para os desempregados, os doentes, os incapacitados, os velhos e os viúvos. BLAND 4 .

O Direito de viver não deve ser interpretado como somente a ampliação da quantidade de anos a serem vividos por um indivíduo, mas, este direito se configura num conjunto de necessidades básicas de caráter biológico, psicológico e social que devem ser atendidas. Na literatura consultada identificou-se diversas formas de apresentar as necessidades e direitos dos seres humanos com idade mais avançada. HORTA ${ }^{12}$ apresenta as necessidades humanas básicas segundo a linha de MASLOW, que podem ser enfocadas agrupadas o u não, projetadas para indivíduos o u grupos populacionais. Neste trabalho optou-se por apresenta-las de modo agrupado e para o grupo populacional de maiores de 60 anos considera-se que mais fortemente estejam interrelacionadas.

A satisfação das necessidades básicas está na dependência do poder de compra ou conquista no mercado social que o indivíduo tenha. Este poder de compra é representado pela força de trabalho que o indivíduo tenha ou seja, sua participação na reprodução do capital. Tanto é assim, que se verifica empiricamente na sociedade contemporânea que as pessoas com mais de 60 anos são marginalizados do sistema de produção, antecedida por um processo gradativo de diferenciação em que as pessoas com menos idade encontram "mais facilmente" trabalho. Nos países em desenvolvimento, há uma crônica satisfação parcial das necessidades do ser humano, havendo seu enfraquecimento gradativo não só porque a idade avança, mas principalmente, porque o processo explorativo o enfraquece, a sociedade em vez de compensá-lo para que ele tenha melhores dias com a capacidade que lhe resta, o marginaliza.

Para THOMAS16 "há uma mitologia segundo a qual as pessoas mais velhas não podem continuar trabalhando depois de atingir certa idade cronológica específica. Embora haja variaçōes de país para país, a remoção compulsória da força trabalhadora depois de 65 anos de idade é uma condição social do envelhecimento na maioria deles. Isso está relacionado com outro valor que tem posição central em algumas sociedades. Trata-se da equiparação da produtividade econômica ao valor pessoal. As pessoas idosas, às quais é sonegado o direito de continuar sendo economicamente recompensadas por seu trabalho, são denegridas como se se tratasse de indivíduos incapazes de desempenhar papéis socialmente produtivos".

Um dos direitos portanto do ser humano que lhe permite viver atendendo as suas necessidades básicas é o trabalho, quando o tem e perde-o legalmente aos 65 anos de idade, quando vive até esta idade. CHEBOTARYOV7 afirma "os indivíduos que, por uma ou outra razão, se viram forçados a se aposentar sofrem da chamada doença dos pensionistas, vale dizer, acentuando declínio da vitalidade, insatisfação com sua condição e, conseqüentemente, envelhecimento prematuro. A ocupação socialmente útil, por outro lado, permite às pessoas mais velhas manter seus papéis anteriores na sociedade e revigorar seu moral".

Outro direito do ser humano, como necessidade básica e que a o idoso, mais que a o jovem, em termos de controle social, existe, é a negação da sexualidade. Ao idoso se por um lado existe a necessidade, a sociedade lhe nega o direito, porque os mitos são múltiplos, a exemplo da exposição do corpo envelhecido, quando deve ser contido porque a função social precípua do sexo é a reprodução. BURNSIDE 5 ; LEA ${ }^{13}$.

\section{COMPROMISSO SOCIAL DA ENFERMAGEM COM O IDOSO}

Considera-se básico para discutir o compromisso social da enfermagem com o idoso, a diferenciaçào entre envelhecimento individual e envelhecimento de populações humanas. Segundo THOMAS 16 "o envelhecimento individual põe em destaque etapas irreversíveis do ciclo de vida, cuja compreensão depende do conhecimento dos processos biológicos, sociológicos e psicológicos associados ao crescimento, amadurecimento e possível declínio do organismo humano, no contexto de ambiente físico, psicológico e social". O envelhecimento de populaçōes humanas, segundo este autor "constitui um fenômeno mais complexo, em relação ao qual pouco consenso existe no que se refere a definição. Grande parte da literatura existente até agora, ao definir o envelhecimento social, põe em desta- 
Souza M.L. e Colaborador - População dos Estados da Região Sul do Brasil e o Compromisso Social da Enfermagem com o Idoso. Rev. Bras. Enf.; RS.36: 192 - 199, 1983.

que o incremento proporcional do número de pessoas mais velhas dentro de cada população". Esta definição de envelhecimento populacional é bastante limitada, em especial se atentarmos aos registros de COWGIL e HOHUES referidos por BASTIAN ${ }^{2}$ que citam terem os idosos, onde forem, feiçōes de minoria em comparação com outros grupos etários.

A forma de conceber a velhice tem sido muito discutida, especialmente pela controvérsia existente entre envelhecimento e os processos patológicos. O presente trabalho aceita o pressuposto de THOMAS ${ }^{16}$, o qual diz "o que se sabe é que o envelhecimento é um processo de diferenciação, tanto do organismo quanto entre seres humanos".

Os trabalhos consultados quer teóricos ou relato de experiência da enfermagem com idosos, se apresentam na linha do envelhecimento individual, mesmo que alguns conceitos permitam visualizar perspectiva de estudos com envelhecimento de populações humanas. Segundo BASTIAN2, "a enfermagem geriátrica preocupa-se com a detecção das necessidades de enfermagem de pessoas idosas, com o planejamento e a implementação da assistência de enfermagem, a fim de atender a estas necessidades e com a avaliação da eficácia e eficiência desta assistência em conseguir manter um nível de bem estar consistente com as limitaçōes impostas pelo envelhecimento". Para HORTA12 "os adultos idosos constituem para a enfermagem grupo etário vulnerável e que esta vulnerabilidade é decorrente de condições físicas, psicológicas e sociais".

O que chama atenção nos conteúdos da literatura consultada, é o direcionamento da assistência de enfermagem a clientela de idosos internados em instituições. Acredita-se que isto é um ponto básico que deva ser questionado, por ter em seu conteúdo alguns aspectos contraditórios. Primeiro, porque centra a assistência de enfermagem em idosos internados em instituições, quando empiricamente se sabe que cerca de dois terços da população de idosos vive em comunidades abertas, em convívio com os demais grupos etários e não somente em instituições quer de caráter hospitalar ou não; em especial porque os serviços de saúde por questões políticas e econômicas não lhe atribuem prioridade. Contraditório ainda, porque individualiza a assistência ao "idoso institucional" mas não aprofunda estudo dos determinantes sociais - políticos e econômicos da condição de envelhecimento de populações humanas.

Necessário se faz relembrar que a internação institucional por diversas razões pode ocorrer, mas não se poderia deixar de registrar a que se segue. Quando as necessidades básicas sucessivamente não são satisfeitas e outras decorrentes da condição orgânica se somam, o indivíduo culmina doente, com problemas que o levam a buscar instituições de saúde. Instituições de saúde estas, cuja acessibilidade muito fortemente é determinada pelo poder aquisitivo. Rompida a barreira seletiva das instituições assistenciais, o velho se defronta com serviços, os quais agravam sua perda de identidade. Ao "velho visível" como mecanismo de compensação ou outro, se presta uma assistência superprotetora e na ânsia de assisti-lo o anula como pessoa, dona de seu corpo e do seu destino, reduzindo-o à condição de objeto da assistência. TIMIO17, a este respeito diz que os progressos da ciência médica tem representado um notável salto na qualidade tecnológica e estrutural, o qual não pode comparar-se com um progresso análogo das relações humanas no interior dos serviços de saúde. As resoluções técnicas propostas pelos descobrimentos científicos criam condições assistenciais alienantes e desumanas, que o isolam de seu ambiente familiar, social e com frequeência dos médicos e enfermeiros.

Indispensável também é verificar como ocorre a internação do velho em instituições, pois esta pode se apresentar como solução aliviadora da família, em especial em internações prolongadas, pois na teoria busca-se dar melhor assistência ao idoso quando na essência busca-se o alívio do encargo. Dependendo da gravidade do caso, ocorre a morte do velho, em leito institucional cuja tecnologia busca a alta qualidade, mas sufoca a essência do ser no direito de ir em busca do outro lado de suas crenças, afastado de sua família, muitas vezes em passado não remoto, razão central de suas lutas sociais.

Estes questionamentos, permitem ainda afirmar que a enfermagem geriátrica ou gerontológica, segue a mesma tendência da enfermagem geral que centra suas atividades em hospitais, o que na verdade é determinado pelo Sistema Nacional de Saúde e que obviamente tem seus determinantes. Assim, a enfermagem geriátrica e/ou gerontológica, além de sofrer a influência dos tempos modernos da enfermagem no Brasil, é dirigida tanto pelo Sistema Nacional de Saúde como pelo sistema político econômico, que privilegia "o homem em idade produtiva".

Considera-se portanto, fundamental que a enfermagem realize uma reflexão de sua prática e também acerca do envelhecimento de populações humanas. É necessário que faça um efetivo preparo dos recursos humanos para que continuamente critique seu trabalho e nesta área, não substime a capacidade de decisão e de auto cuidado do idoso, especialmente quando uma de suas funções vitais estiver comprometida. Isto porque, empiricamente se vê no campo de prática a enfermagem dispensando cuidados que muito bem poderiam ser realizados pelo cliente e/ou familiar, mas que provavelmente pelo caráter "paternalista/maternalista" da enfermagem, ou mesmo o "monopólio do saber-cuidar", ainda o auto cuidado e a valorização da auto determinação do idoso não é um fato concreto na prática, muito embora seja enfocado teoricamente. 
Souza M.L. e Colaborador - População dos Estados da Região Sul do Brasil e o Compromisso Social da Enfermagem com o Idoso. Rev. Bras. Enf.; RS.36: 192 - 199, 1983.

CAMPEDELLI ${ }^{6}$, no relato de sua experiência com idosos, chama atenção que a realização de cursos com temática voltada para enfermidades que mais os afligiam, foi inteiramente frustada porque o grupo passou a ter os sintomas das doenças abordadas no curso. Assim, a atividade com os idosos passou a ser centrada no trabalho artesanal e com voluntariado e terapeuta ocupacional, trabalho permanente de uma enfermeira de saúde pública e uma visitadora, além do trabalho periódico de um psiquiatra, determinado pela necessidade deste grupo.

Este é um exemplo típico de grande esforço da enfermagem, mas que reflete a sua prática de mediadora e não de agente de mudança. A medida em que se atender pequenos grupos de modo isolado, socialmente se lhes contorna ou controla tensões, se dá aproveitamento do idoso às suas aptidões de trabalho (não lucrativo pessoal), mas de uma forma limitada onde a responsabilidade do Estado permanece no discurso mas não na prática.

Reconhecemos que uma mudança da prática da enfermagem em muito contribuirá para maior conforto e satisfação dos que atuam nesta área e dos idosos assistidos, mas a situação social dos idosos só será mais digna com uma mudança mais ampla na sociedade, na dimensão econômica, política, educacional e cultural.

\section{CONSIDERAÇÕES ALTERNATIVAS}

A humanidade não é uma espécie animal: é uma realidade histórica. BEAUVOIR ${ }^{3}$. Acreditando que o presente trabalho possa servir de subsídio à enfermagem, para maior enfrentamento com a realidade social que é o seu campo de prática, conclui-se ser necessário: 1) participar do processo de valorização do ser humano, estudando seu ciclo vital se engajando num processo de educação social buscando não só evidenciar os problemas existentes, mas participando da busca de soluções alternativas; 2) reavaliar a prática da enfermagem de modo que a assistência não seja mais um instrumento de anulação do ser humano em idade avançada e sim favoreça sua libertação dos mitos impostos. Como sugere THOMAS16 "precisamos examinar as atividades e suposiçōes culturais subjacentes a respeito do envelhecimento, verificando, inclusive, como podem ser elas modificadas a fim de proporcionar um enfoque mais realista e significativo do envelhecimento na sociedade".

\section{SUMMARY}

The authors attempt to consider some theorical points of view related with social matters of old-age individuals in terms of population.

Also, they intend to establish some comparative parameters between age groups less than 20 years and older than 60 years, trying to emphasize the southern population of Brazil, (Santa Catarina, Paraná and Rio Grande do Sul) within this context.

The authors discuss some concepts in which they establish some basis for nursery assistance and they criticize its practice in relation to assistance in older people. They conclude their basis saying that Nursery should have a very important roll participating in the social education process searching for an increase in human being valorization especially older people. Furthermore, they consider the need for a new assessment of practice in Nursery, so assistance cannot be used as a tool for human being segregation for oldage people, but as an instrument that can lead to wipe out some social barriers and long-standing myths.

\section{REFERÊNCIAS BIBLIOGRÁFICAS}

1. BARROS F., M. A. de. Contribuição ao estudo da mortalidade em Santa Catarina. 1979, Florianópolis, SC (Tese de Concurso para Professor Titular da UFSC).

2. BASTIAN, E. M. Gerontologia: campo de enfermagem. Enf. Novas Dimensões, 5 (2): 17-22, 1979.

3. BEAUVOIR, S. O segundo sexo. Fatos e mitos. Editora Nova Fronteira. Rio de Janeiro, 1980.

4. BLAND, J. A terceira idade. A saúde no mundo, 12-15, abril 1979. (publicação da OMS).

5. BURNSIDE, I. M. Enfermagem e os idosos. Organização Andrei Editora, São Paulo, 1979.

6. CAMPEDELLI, M. C. Assistência de Enfermagem de Saúde Pública a um grupo de velhos. Relato de experiência. Enf. Novas Dimensões. 4 (2): 86-89, 1978.

7. CHEBOTARYOV, D. Gozando a velhice. A saúde do mundo, 8-11, abril 1979 (publicação da OMS)

8. COSTA, R. V. da. A velhice no mundo e no Brasil. Aspectos demográficos. A qualidade da vida. O papel do Estado e da iniciativa privada. Femina, : 863-70, Novembro 1978 .

9. FUSTINONI, O. A terceira idade: desafios e oportunidades. Contact 22: 4-5, 1982. 
Souza M.L. e Colaborador - População dos Estados da Região Sul do Brasil e o Compromisso Social da Enfermagem com o Idoso. Rev. Bras. Enf.; RS.36: 192 - 199, 1983.

10. FIBGE. Recenceamento geral do Brasil, Rio Grande do Sul, Santa Catarina e Paraná 1960, 1970 e 1980.

11. FIBGE. Indicadores Sociais. Tabelas Selecionadas. 1979.

12. HORTA, W. de A. A assistência de Enfermagem ao Adulto Idoso. Enf. Novas Dimensões. 4 (5): 268-73, 1978.

13. LEA, M. Quem tem medo de envelhecer? Rio de Janeiro, Record, 1981.

14. QUADRA, A. A. F. et alii. Marcos conceituais do atendimento integral a o idoso. RPH 29 (1): 3-8, 1981.

15. SVANBORG, A. A idade avançada e em boa forma. A saúde no mundo. 16-19, abril 1979 (publicação da OMS).

16. THOMAS, R. G. Os idosos num mundo em transformação. A saúde no mundo. 3-7, abril 1979 (publicação da OMS).

17. TIMIO, M. Classes sociales y enfermedad. Introducción a una epidemiologia diferencial. Editorial Nueva Imagen. México. DF. 1980. p. 121.

18. VIEDMA. C, Um desafio mundial. A saúde no mundo. 20-22, abril 1979 (publicação da OMS).

\section{AVALIAÇÃO DE UM PROGRAMA DE ASSISTÊNCIA PRIMÁRIA DE SAÚDE NA ÁREA PERIURBANA}

* Rosana Lúcia Alves de Vilar

** Mary Anne Small

$\mathrm{ReBEn} / 09$

Vilar R. L. A. e Colaboradora - Avaliação de um Programa de Assistência Primária de Saúde na Área Periurbana Rev. Bras. Enf.; RS 36: 199 - 212; 1983

\section{RESUMO}

Foi implantado e executado um Programa de Assistência Primária de Saúde em duas comunidades da área periurbana da cidade de Natal-RN. A finalidade deste programa foi de elevar o nível de saúde da comunidade, e seu elemento-chave era a participação comunitária através, principalmente, do trabalho da promotora de saúde. Será detalhado o desenvolvimento do programa e apresentada uma avaliação baseada em mudanças mensuráveis de práticas de saúde e utilização dos serviços de saúde, como observado nestas comunidades no período de dois anos.

\section{INTRODUÇÃO}

O Estado do Rio Grande do Norte vem sofrendo um processo de urbanização crescente. No censo de 1970 , a população urbana foi de $51,16 \%$, e, em 1980 , com uma população total de 1.899 .725 habitantes, este percentual subiu para 58,73\%. A taxa de crescimento populacional do ano de 1980 ainda mostra, com bastante evidência, este aumento populacional, que, para todo o Estado, foi de 2,04\% e, para a capital - Natal, com 416.906 habitantes, foi de 4,65\%. para ser mais explícito, se o crescimento continuasse neste ritmo, em 34 anos a população do Estado duplicaria, enquanto que isto ocorreria, na capital, em 15 anos. (SINOPSE RN-1980).

* Professora Assistente do Departamento de Enfermagem, Universidade Federal do Rio Grande do Norte, Brasil.

**Enfermeira Educadora de Saúde Comunitária do Projeto HOPE, em Millwood, Virginia, E. U. A., Professora Visitante do Departamento de Enfermagem da Universidade Federal do Rio Grande do Norte de 1974-1981. 